\title{
CYP3A4 activity and haloperidol effects in alcohol addicts
}

\author{
M.S. Zastrozhin ${ }^{\mathrm{a}, \mathrm{b}, *}$, V.V. Smirnov ${ }^{\mathrm{c}}$, D.A. Sychev ${ }^{\mathrm{a}}$, L.M. Savchenko ${ }^{\mathrm{a}}$, E.A. Bryun ${ }^{\mathrm{b}}$ and O.A. Matis ${ }^{\mathrm{b}}$ \\ ${ }^{a}$ Russian Medical Academy of Postgraduate Education, Moscow, Russia \\ ${ }^{\mathrm{b}}$ Moscow Research and Practical Centre for Narcology of the Departament of Public Health, Moscow, \\ Russia \\ ${ }^{\mathrm{c} N a t i o n a l}$ Research Center - Institute of Immunology, Federal Medical-Biological Agency of Russia, \\ Moscow, Russia
}

*Corresponding author. E-mail: rudnmed@yandex.ru

\begin{abstract}
BACKGROUND: Haloperidol is one of the most commonly used typical antipsychotics [2]. It has a powerful antipsychotic activity blocking mesolimbic postsynaptic dopamine receptors. Unwanted adverse effects accompany the use of haloperidol. Therefore alcohol abusers' attitudes towards haloperidol are ambiguous and often negative, which sometimes limits it's use in patients with addictive disorders [3]. Cytosolic carbonyl reductase reduces haloperidol to reduced form, which has $10-20 \%$ of the activity of the parent molecule. It is further metabolized by CYP3 A4 to a tetrahydropyridine and then conjugated by glucuronidation and sulphation. Reduced haloperidol is back-oxidized to haloperidol by CYP3A4 and CYP2D6. Haloperidol is N-dealkylated by CYP3A4 and CYP2D6 to 4-chlorophenyl-4hydroxypiperidine and p-fluorobenzoyl propionic acid. The correlation between CYP2D6 and CYP3A4 activity and the rate of biotransformation of haloperidol was demonstrated in a number of studies on patients with schizophrenia $[1,2,5]$. At the same time other studies deny or disaffirm this correlation [4].
\end{abstract}

OBJECTIVE: To estimate the correlation between CYP3A4 isoenzyme activity and the efficacy and safety of haloperidol in patients with alcohol abuse during the exacerbation of the addiction.

METHODS: The study involved 15 men, alcohol abusers, in exacerbation of their addiction, who were hospitalized in Moscow Research and Practical Centre for Narcology of the Departament of Public Health. All 15 patients received haloperidol in tablets and injections. Determination of CYP3A4 activity was performed using high performance liquid chromatography with mass spectrometry (HPLC/ MS) by determination of endogenous substrate of this isoenzyme and its metabolite in urine - the ratio: cortisol/6beta-hydroxycortisol. We used international psychometric scales to assess efficacy of haloperidol (the scale of determining the severity of addiction of The National Research Center on Addictions of the Ministry of Health Of Russia, Hamilton Anxiety Research Scale (HARS)). The safety of haloperidol was estimated by the UKU Side-Effect Rating Scale. Scales express the clinical picture of the abuse. The higher the score, the more pronounced the addiction is. Calculating the differences in scores of the scales allowed for clinical assessment of haloperidol effects. The larger the difference in scores was, the more pronounced were the changes in clinical picture of abuse, and the higher the efficacy of therapy was assumed. Statistical analysis of the results of the study was performed by non-parametric statistics by the program STATISTICA v10.0 («StatSoft Inc.», USA). The normality of sample distribution was estimated by Shapiro-Wilk's W-test, and the homogeneity of dispersion, that was estimated by Fisher's 
T-test (in case of comparison of two samples). The differences were evaluated as significant in case of $p<0,05$ (statistic power $>80 \%$ ). To determine the correlation between the quantitative characteristics Spearman rank R coefficient was calculated. The value of correlation coefficient $r$ from 0,3 to 0,7 $(p<0,05)$ indicated positive moderate, but significant correlation between the characteristics, $r>0,7$ $(p<0,05)$ - strong and significant correlation, negative value of $r$ indicated inverse correlation.

RESULTS: Data analysis demonstrated a correlation between the activity of isoenzyme CYP3A4 and the scores of pathological addiction $\left(r_{1}=-0,36\right)$, HARS $\left(r_{2}=-0,45\right)$, UKU Side-Effect Rating Scale $\left(r_{3}=\right.$ $-0.15)$ in the entire group $(p<0.05)$. In a group of patients, who received the higher doses of haloperidol (more than $7.5 \mathrm{mg}$ per day in tablets or $5 \mathrm{mg}$ per day in injections), the following results were received in the same groups of data: $r_{1}=-0.68, r_{2}=-0.71, r_{3}=-0.76(p<0.05)$.

CONCLUSION: The results demonstrate the correlation between CYP3A4 activities and the efficacy and safety of haloperidol in alcohol abusers during the exacerbation of the addiction. The inverse correlation indicates that the higher the activity of CYP3A4 is, the lower the efficacy of haloperidol is. Also it can be assumed that the presence of strong correlation between the activity of CYP3A4 and the efficacy of haloperidol in group of patients, who received higher doses of haloperidol, may indicate that CYP3A4 is involved in haloperidol metabolism when it is used at higher doses.

Limitations of the study: It should be noted that in this research the activity of CYP3A4 was determined using high performance liquid chromatography with mass spectrometry (HPLC/MS) by determination the ratio of cortisol/6-beta-hydroxycortisol for the fist time. To increase the level of our confidence in the results further studies with a larger number of people are necessary.

Keywords: Haloperidol, biotransformation, CYP3A4, ABCB1, side effects, alcohol abuse

\section{Conflict of interest statement: None.}

\section{References}

[1] Butwicka A1, Krystyna S, Retka W, Wolańczyk T. Neuroleptic malignant syndrome in an adolescent with CYP2D6 deficiency. Eur J Pediatr. 2014;173(12):1639-42. doi: 10.1007/s00431-013-2208-z. Epub 2013 Nov 20.

[2] Gasso P, Papagianni K, Bobadilla RF, Arnaiz JA, Bernardo M, Lafuente A. Relationship between CYP2D6 genotype and haloperidol pharmacokinetics and extrapyramidal symptoms in healthy volunteers. Pharmacogenomics. 2013;14(13):155163. doi: $10.2217 /$ pgs. 13.150

[3] Ju.P. Sivolap, Rational approaches to the use of antipsychotic drug treatment in practice. "Zdorovaja Ukraina" 7/1, 2008, p. 83-84 (in Russian).

[4] Nakamura A1, Mihara K, Ne moto K, Nagai G, Kagawa S, Suzuki T, Kondo T. Lack of correlation between the steadystate plasma concentrations of aripiprazole and haloperidol in Japanese patients with schizophrenia. Ther Drug Monit. 2014;36(6):815-8.

[5] Van der Weide K1, van der Weide J. The Influence of the CYP3A4*22 Polymorphism and CYP2D6 Polymorphisms on Serum Concentrations of Aripiprazole, Haloperidol.- 